\title{
Application of Neural Networks on Off-Road Vehicles
} m Watany, M Mahmoud, H.A Zaffan

\begin{abstract}
Off-road vehicle traction performance has been considered as an important point of research for many years theoretically and experimeiitally, where the design of traction device system requires developments of mathematical models. One of these developments is directed to predict the performance of vehicle traction. Recently, Artificial Netiral Networks (ANN's) seems to provide an alternative approach to the modeling of off-road vehicle performance. In this paper, an application used for computing the drawbar-pull for off-road vehicle as a function of some input set of data vectors using a suggested ANN's model is considered. The suggested model provided with more simplified topology of ANN's architecture. The model comprises only a single hidden layer of neurons with some dynamic controllability by monotonous increasing of hidden layer neuron number. Comparisons between the suggested model results and others proving the better performance of the simplified model. However the relationships between pull and load at different running conditions (inflation pressure, tyre size, and pull angle) have been predicted using the ANN's model. Comparisons between previous model and published data with ANN's predicted results have been made and the agreement seems to be good. The model presented herein is a multi input-one hidden layerone single output, and suggestion for fulier development is, th result model. Finally, a suggested direction of fhture, modification of our model with more biologically plausible consideratiqn is given. The expected advantage for such modified models is that they are more practically applicable to solve complex engineering problems in the real world
\end{abstract}

